\title{
Modeling and protein function prediction of truncated form of geobacillus thermocatenulatus lipase [BTL2]
}

\author{
M.A. Ghafouri ${ }^{\bowtie}$, A.A. Karkhane ${ }^{2}$, M.R. Azimi', B. Yakhchali², N. Goudarzi² \\ 'Department of Plant Breeding, Univercity of Zanjan, Zanjan, Iran \\ ${ }^{2}$ Department of Industry and Enviroment Biotechnology,National Institute of Genetic engineering and Biotechnology, \\ Tehran, Iran
}

\begin{abstract}
Motivations
Lipases, mainly of microbial origin, represent the most widely used class of enzymes in biotechnological applications and organic chemistry. Bacterial lipases are members of the structural superfamily of $\alpha / \beta$ hydrolases that catalyze the hydrolysis and synthesis of a variety of acylglycerols at the lipid-water interface. Optimization of features and catalytic activity of lipases as the third industrial enzyme are significant for industrial applications. The structural comparison between $\alpha / \beta$ hydrolases and thermoalkalophilic lipases represented that the insertion of $\mathrm{Zn} 2+$ binding site $(\alpha 3, b 1$ and b2) has not, until now, been seen within the $\alpha / \beta$ hydrolase canonical fold that making a tight intramolecular interaction with the main catalytic domain. In this study, we investigated the role of $\mathrm{Zn} 2+$ binding site on lipase activity by using the computational methods.
\end{abstract}

\section{Methods}

To surveythe effect of $Z \mathrm{n} 2+$ binding site on structure and function of lipase, the nucleotide sequence of $\alpha 3$ domain was deleted in the Geobacillus thermocatenulatus lipase gene. Homology modeling for the native and the mutated lipases was performed using MODELLERV9.10, based on crystal structure of Geobacillus thermocatenulatus (PDB: 2w22 (opened form)) as tempelate. Analysis of the 3D-structure of the native and the mutated lipases generated by MODELLER were done using QMEAN and ProsA servers. The molecular docking of native and mutated lipases with ligands (Dibutyrin, tributyrin, tricaproin, and tricaprylin) was performed using the trial Molegro Virtual Docker 5.0. Before docking, the structure of receptor and ligands was prepared, flexible torsions in ligands detected, explicit hydrogens crated and possible missing bonds assigned.

\section{Results}

Z-scores of the two lipases were calculated from PROSA-web. It was found to be -8.32 and -8 for the native and mutated lipases respectively. This result confirmed that there is high similarity between native and mutated lipases in opened conformation. Also the QMEAN Server was used for model quality estimation of the lipases. It was found to be 0.833 and 0.749 for the native and chimera lipases respectively. Results obtained by QMEAN Server specify the reliability of the models and also, confirmed that there is high similarity between two lipases. Furthermore, the root mean-squared deviation (RMSD) value of the native and mutated lipases was calculated by superimposing two lipases (388 residues). The RMSD was found to be $12.62 \mathrm{~A}^{\circ}$ for the opened form of the lipases. We carried out molecular docking with a series of substrates. In comparison of mutated lipase interaction energy with BTL2 lipase, the mutated lipase showed lower energy to break down bonds between ligand and lipase. The function prediction of mutated lipase with deletion on $\alpha 3$ domain, represent lipase activity enhancement with $\mathrm{C} 4$ to $\mathrm{Cl0}$ substrates. Therefore function of mutated lipase in hydrolyzing of triacylglycerol is better than BTL2. 